partially inhibited by up to $100 \mathrm{~mm}-\mathrm{NaF}$. With $\beta$-glycerophosphate as substrate, activity in the pellet was $77 \mathrm{nmoles}$ of $P$ liberated $/ \mathrm{hr} . / \mathrm{mg}$. of protein. It was greatly stimulated by $10 \mathrm{~mm}-\mathrm{Mg}^{2+}$ but was unaffected by $\mathrm{Ca}^{2+}, \mathrm{Na}^{+}$or $\mathrm{K}^{+}$; it was inhibited completely by $10 \mathrm{~mm}-\mathrm{NaF}$. The $\mathrm{pH}$ optimum of the pellet enzyme was between $4 \cdot 8$ and $5 \cdot 0$. The supernatant contained no activity. These results were similar when centrifugation was performed at $45000 \mathrm{rev}$. $/ \mathrm{min}$. for $60 \mathrm{~min}$.

It appears that rat heart contains several acid phosphatases, and their subcellular distribution is being investigated.

Chen, P. S., Toribara, T. Y. \& Warner, H. (1956). Analyt. Chem. 28, 1756.

Cleland, K. W. \& Slater, E. C. (1953). Biochem. J. 53, 547. de Duve, Ch. (1963). In Ciba Found. Symp.: Lysosomes, p. 1. Ed. by de Reuck, A. V. S. \& Cameron, M. P. London: J. and A. Churchill Ltd.

Kilsheimer, G. S. \& Axelrod, B. (1957). J. biol. Chem. 227, 879.

Lowry, O. H., Rosebrough, N. J., Farr, A. L. \& Randall, R. J. (1951). J. biol. Chem. 193, 265.

Tappel, A. L., Sawant, P. L. \& Shibko, S. (1963). In Ciba Found. Symp.: on Lysosomes, p. 78. Ed. by de Reuck, A. V. S. \& Cameron, M. P. London: J. and A. Churchill Ltd.

\section{Lysosomes in Hepatomas}

By R. Wattiaux, S. Wattiaux-De Coninck, J. M. VAN DiJCK and H. P. MoRRIs. (Laboratory of Physiological Chemistry, Facultes Universitaires Notre-Dame de la Paix, Namur, Belgium, and Howard University, Washington, D.C., U.S.A.)

Few studies have been undertaken on the characterization of lysosomes of cancer cells. Therefore we started investigating the properties of these subcellular structures in several tumours. Results reported here have been obtained on five transplantable Morris hepatomas: 7316A, 7794A, 7794B, 5123A and HC. Three characteristic aspects of the organelles have been considered: their enzymic content, their sedimentation patterns in iso-osmotic sucrose and their behaviour in isopycnic centrifugation in a sucrose gradient and in a gradient made of glycogen dissolved in aqueous sucrose. The results have been compared with those obtained by de Duve and his co-workers on rat liver granules. The activities of the seven acid hydrolases we have assayed exhibit certain variations from one tumour to another, but are of the same order of magnitude as those found in rat liver. The distribution pattern of the enzymes after differential centrifugation is similar to that recorded for the liver enzymes: the hydrolases are mostly recovered in the mitochondrial fractions and show a peak of specific activity in the light-mitochondrial fraction. The behaviour of acid phosphatase and acid deoxyribonuclease in gradient-centrifugation experiments suggests that the osmotic space of the lysosome is smaller in the hepatoma than in the liver and that the space freely accessible to sucrose is larger.

\section{Latency of Endogenous and Exogenous Lyso- somal Glucosidases}

By J. B. Lloyd.* (Department of Biochemistry, University of Miami, Miami, Fla., U.S.A.)

Free and total maltase, cellobiase, $p$-nitrophenyl $\alpha$-glucosidase and $p$-nitrophenyl $\beta$-glucosidase activities were measured at $\mathrm{pH} 5 \cdot 0$ in lysosome-rich liver fractions prepared from rats pre-injected with glucosidases of plant origin.

Male Wistar rats were starved overnight and injected intravenously with purified Aspergillus niger amyloglucosidase (Qureshi, 1967) (250 mg./kg. body wt.), with almond emulsin $\beta$-glucosidase (Sigma Chemical Co., St Louis, Mo., U.S.A.) (125mg./kg.) or with iso-osmotic saline only. Rats were killed $4 \cdot 5-5 \mathrm{hr}$. after injection, and their livers quickly removed and homogenized in cold 0.25 M-D-mannitol. A large-granule fraction, sedimenting between $11000 \mathrm{~g}$-min. and $225000 \mathrm{~g}$-min., was prepared, resuspended in cold mannitol and assayed immediately for free and total glucosidases.

Six rats were injected with the fungal amyloglucosidase $(A)$, five with emulsin $\beta$-glucosidase $(B)$ and five with saline. Maltase activity in granules from rats given $A$ was approximately three times that in those from rats given $B$ or saline. However, the proportion of free to total maltase was the same $(A$, mean $10.4 \% \pm$ s.D. $4 \cdot 1 \% ; B, 10 \cdot 6 \% \pm$ s.D. $4.7 \%$; saline, $10 \cdot 7 \% \pm$ s.D. $2.9 \%$ ), suggesting that injected $A$ reaches the interior of liver lysosomes. This is consistent with the observed effect of the enzyme in depleting intralysosomal glycogen in Pompe's glycogenosis (Hug \& Schubert, 1967).

Injections of $A$ could simply have raised the endogenous maltase activity. However, $p$-nitrophenyl $\alpha$-glucosidase activities were not significantly higher in the lysosomes of $A$-injected rats. The additional maltase activity that followed injection of $A$ must, therefore, be due to an enzyme relatively more active on maltose than on $p$-nitrophenyl $\alpha$-glucoside. Additional experiments showed that this corresponds to the substrate-specificity of $A$.

Beck \& Tappel (1968) showed that the $p$-nitro-

* Present address: Department of Biochemistry, University College, Cardiff. 